weight, preterm birth and neonatal morbidity and mortality. In turn, these outcomes are likely to result in increased well-being of this vulnerable group of women. Such interventions may have to be delivered opportunistically and psychiatrists may be best placed to do so. Therefore there is an onus on each of us to consider the physical well-being of our patients during pregnancy and to offer advice and education regarding smoking and other drug use.

\section{Declaration of interest}

None.

\section{References}

COLEMAN,T. (2004) Special groups of smokers. BMJ, 328, 575-577.

HOWARD, L. M., KUMAR, C., LEESE, M., et al (2002) The general fertility rate in women with psychotic disorders. American Journal of Psychiatry, 159, 991-997

HOWARD, L. M., GOSS, C., LEESE, M., et al (2003) Medical outcome of pregnancy in women with psychotic disorders and their infants in the first year after birth. British Journal of Psychiatry, 182, 63-67.

MAUGHAN, B., TAYLOR, A., CASPI, A., et al (2004) Prenatal smoking and early childhood conduct problems: testing genetic and environmental explanations of the association. Archives of General Psychiatry, 61, $836-843$

McCREADIE, R. G. (2002) Use of drugs, alcohol and tobacco by people with schizophrenia: case-control study. British Journal of Psychiatry, 181 321-325.

SACKER, A., DONE, D. J. \& CROW, T. J. (1996) Obstetric complications in children born to parents with schizophrenia: a meta-analysis of case-control studies. Psychological Medicine, 26, 279-287.
*Nisha Shah Specialist Registrar, North Islington Drugs Service, 592 Holloway Road, London N7 6LB, e-mail: nisha.shah@candi.nhs.uk, Louise Howard Senior Lecturer inWomen's Mental Health, Health Services Research Department, Institute of Psychiatry, London SE5 8AF

\title{
Visual acuity and reported eye problems among psychiatric in-patients
}

\section{AIMS AND METHOD}

The aim of this study was to examine visual problems among patients admitted to an inner city acute mental health unit. We measured visual acuity using a Snellen chart. Patients were also asked about perceived eye problems and access to services.

\section{RESULTS}

Of 55 in-patients on five acute general adult wards at an inner city mental health unit over a 3-day period, 31 agreed to participate in the study. Twenty $(65 \%)$ had impaired visual acuity and $19(61 \%)$ had not been to an optician for 5 or more years. Seventeen patients (55\%) reported experiencing difficulty with their eyesight. The main problems reported were blurring of vision and periorbital pain. Of these 17 patients, $15(88 \%)$ had impaired visual acuity on Snellen testing. Half of those who had previously been prescribed

\begin{abstract}
glasses or contact lenses reported that they had been lost.
\end{abstract}

\section{CLINICAL IMPLICATIONS}

Visual impairment appears to be another area of physical health which is underrecognised and undertreated in people with severe mental health problems. Although there are numerous issues that must be addressed by mental health staff, patients should be asked about eye problems and supported in accessing opticians.
There is substantial evidence that people with severe mental illness have poorer physical health than the general population and increased mortality from natural causes (Harris \& Barraclough, 1998). Few studies have specifically examined eye problems in people with severe mental illness. Two studies, conducted outside the UK, detected high rates of eye disease and untreated visual acuity problems (Smith et al, 1997; Ungvari et al, 2002). Furthermore, despite well recognised ocular side-effects of psychotropic medications, there are few data on the frequency with which these medications routinely cause visual impairment in psychiatric patients (McCarty et al, 1999).

Many of the factors known to have a detrimental effect on the physical health of people with severe mental illness (diet, alcohol consumption, lack of exercise and smoking) also affect vision. Moreover, common conditions such as diabetes mellitus and ischaemic heart disease are more common in this group than in the general population - and people with severe mental illness are less likely to access appropriate services.

To identify the incidence of impaired visual acuity we conducted a cross-sectional descriptive study of psychiatric in-patients with severe mental illness and collected subjective information about eye problems and access to services.

\section{Method}

In-patients from five acute general adult wards at an inner city mental health unit were invited to participate in the study. Patients who were acutely intoxicated or 
agitated were excluded, as were those who could not speak or read English. All other in-patients aged between 17 and 65 who were present in the hospital over a 3-day original papers period were invited to participate. Two wards were covered on each of the first 2 days and the last ward on the third day.

An information sheet was given to patients to explain the purpose of the research and what it involved. Patients were asked to sign a consent form if they agreed to participate. Data collected included name, age, diagnosis, medication and details about prescribed glasses or contact lenses. Each patient was then asked a number of standard questions - if/when they had last visited an optician, if any professionals had asked about their eyesight before and if they had noticed any problems with their vision. Data were stored in a secure place at all times during the study and only those involved in the study had access to the data at any time. The study was approved by the Riverside Research Ethics Committee. Confidentiality was ensured at all times.

The methodology was devised in conjunction with ophthalmologists and the optician from the general hospital. Visual acuity was tested using a Snellen chart, which consists of lines of letters which decrease in size when moving from the top to the bottom of the chart (as used by opticians). The chart was placed at eye level at a distance of $6 \mathrm{~m}$ from the patient in a well-lit room and an occluder was used to test each eye separately. Patients who had spectacles or contact lenses were instructed to read the Snellen chart first without their glasses/contact lenses and then with them on. A visual acuity of $6 / 9$ or below was considered 'impaired'.

Patients with impaired visual acuity were asked to read the Snellen chart again, this time using a pinhole to determine if they had a refractive or a non-refractive error. If visual acuity improved with pinhole correction, this indicated a 'refractive error' (glasses would correct the impairment). If acuity did not improve with the pinhole, this indicated a 'non-refractive error' (impaired visual acuity would not improve with glasses).

\section{Results}

Of 55 patients who were invited to participate, 18 (33\%) refused, 4 (7\%) were too disturbed to be interviewed and 2 (4\%) could not speak or read English. The remaining 31 patients (56\%) agreed to participate in the study. Of the 18 patients who refused, 13 did not give a reason and 5 considered their eyesight was fine and did not feel that they needed an eye test. There were no significant differences in the age, gender or ethnicity of those who refused and those who participated. Eleven of those patients (61\%) who refused had been admitted within the past 7 days and were acutely unwell and in some cases agitated. Of the 31 patients who agreed to participate, $5(16 \%)$ had been admitted within the past 7 days, $7(23 \%)$ within the past month, $14(45 \%)$ had been in hospital for between 1 and 3 months and 5 (16\%) for between 3 and 6 months. The mean age of the sample was 37 years, with an almost equal male to female ratio
Table 1. Visual impairment and reported eye problems among

in-patients with severe mental illness $(n=31)$

$n(\%)$

Optician visit within 5 years

Yes

$12(39)$

No

$19(61)$

Reported eye problems

Yes

$17(55)$

No

Measured visual acuity

No significant visual impairment 11 (35)

Visual acuity $6 / 9$ or less

$20(65)$

Types of visual impairment $(n=20)$

Bilateral

$14(70)$

Unilateral

6 (30)

Refractive error

$12(60)$

Non-refractive error

$8(40)$

of $15: 16$. Most $(21,68 \%)$ were of White British or European origin with 8 (26\%) of Black British/AfricanCaribbean origin and $2(6 \%)$ of 'other' ethnic origin. Of the 31 patients tested, 13 (42\%) had ICD-10 diagnoses of schizophrenia or another psychotic illness (F20-29), 6 (19\%) had a depressive illness (F32-39), 5 (16\%) had bipolar affective disorder (F31) and 4 (13\%) had mental and behavioural disorders owing to substance misuse (F10-F19) (World Health Organization, 1993). Three patients (10\%) had not yet received a clinical diagnosis.

Of the 31 patients tested, two-thirds had impaired visual acuity of $6 / 9$ or below (Table 1 ). Of the 17 patients who reported experiencing difficulty with their eyesight, 15 (88\%) had impaired visual acuity on Snellen testing. The main problems reported included blurred vision, headaches and periorbital pain. Eight patients tested (26\%) had previously been prescribed glasses or contact lenses, but half (4) patients had lost them.

All participants considered their eyesight to be important and 27 (87\%) felt it important to visit an optician every 2 years for a routine check-up. However, 19 (61\%) reported that they had not been to an optician in 5 or more years. The reasons given were that it had not occurred to them, they had considered seeing an optician for a routine visit but did not go as they felt their eyesight was fine, they had been too unwell with their psychiatric illness or 'laziness'. Two patients said they had never visited an optician in their life. Of the 31 patients tested, 5 (16\%) reported that a professional had previously asked them about their eyesight; 2 of these 5 had received treatment from an ophthalmologist in the past for eye problems such as cataracts. The other 3 said that their general practitioner had asked about their eyesight in the past.

Two patients had insulin-dependent diabetes mellitus and 3 had hypertension; 4 of these 5 patients had impaired visual acuity; 2 of these patients had impaired acuity with a non-refractive error on Snellen testing.

Twenty-four participants (77\%) were on antipsychotic medication at the time of testing and 7 were known to have been on antipsychotics for 5 or more 
years. There may have been more patients who had been on antipsychotic medication in the long term but it was not possible to obtain this information in several cases. Of the 7 patients known to be on antipsychotics for 5 or more years, 5 had impaired visual acuity.

\section{Discussion}

About two-thirds of those psychiatric in-patients tested had impaired visual acuity. It is not possible to compare this figure with that for the general population, as data on prevalence of visual impairment in the general population are scarce (Hayward et al, 2002), but our patients visited opticians less frequently (Department of Health, 2003). These results are similar to those described in studies from Hong Kong (Ungvari et al, 2002) and Australia (Smith et al, 1997), in which $75 \%$ of chronic inpatients and $70 \%$ of in-patients with schizophrenia had impaired visual acuity respectively. The Australian study also showed a high prevalence of ocular abnormalities, including cataracts and corneal pigmentation.

The main limitations of our study are its crosssectional design, the small sample size and the fact that it was conducted on one site. In addition, we were unable to conduct comprehensive eye examinations, including fundoscopy, which would have revealed cataracts or glaucoma in some patients, particularly those found to have non-refractive errors.

The findings of our study supported the recent American Psychiatric Association guidelines (2004) on physical and laboratory assessments for patients with schizophrenia, which recommend ocular examination every 2 years for patients under 40 and every year for patients over 40 .

Our results are consistent with other findings of physical ill health in psychiatric patients (Harris \& Barraclough, 1998) and have implications for routine clinical practice and service development. It would be beneficial for mental health professionals to consider eyesight and eye health when assessing patients for the first time on admission. Staff should ask about eye problems and the prescription of glasses/contact lenses as part of the routine assessment of physical health; if glasses and/or contact lenses have been lost, staff should help in obtaining new ones. It may be feasible for junior doctors to use a Snellen chart on the ward to assess the visual acuity of some patients who report problems with their vision. Consideration should also be given to establishing links between mental health services and opticians to facilitate access for vulnerable patients.

\section{Declaration of interest}

None.

\section{References}

AMERICAN PSYCHIATRIC

ASSOCIATION (2004) Practice Guidelines for theTreatment of Patients psychotropic medication, and cataract. with Schizophrenia (2nd edn). Washington, DC: APA.

DEPARTMENT OF HEALTH (2003) Ophthalmic Statistics for England. London: Department of Health. http:// www.dh.gov.uk/assetRoot/04/08/ 06/75/04080675.pdf

HARRIS, E. C. \& BARRACLOUGH, B. (1998) Excess mortality of mental disorder. British Journal of Psychiatry, $173,11-53$.

HAYWARD, L. M., BURDEN, M. L., BURDEN, A. C., et al (2002) What is the prevalence of visual impairment in the general and diabetic populations: are there gender and ethnic differences? Diabetic Medicine, 19, 27-34. Ophthalmology, 106, 583-687.

SMITH, D., PANTELIS, C., MCGRATH, J., et al (1997) Ocular abnormalities in chronic schizophrenia: clinical implications. Australian and New Zealand Journal of Psychiatry, 31, 252-256.

UNGVARI, C. S., TANG,W. K., WONG,W. K., et al (2002) Distant visual acuity in chronic psychiatric patients. A pilot study. Social Psychiatry and Psychiatric Epidemiology, 37, 488-491.

WORLDHEALTH ORGANIZATION (1993) The ICD-10 Classification of Mental and Behavioural Disorders: Diagnostic Criteria for Research. Geneva:WHO.

Bhaskar Punukollu Specialist Registrar in Adult and Community Psychiatry, Charing Cross and St Mary's Higher Specialist Training Scheme, London, *Michael Phelan Consultant Psychiatrist, West London Mental Health NHS Trust, Gloucester House, 194 Hammersmith Road, LondonW6 8BS, e-mail: michael.phelan@wlmht.nhs.uk original papers 